\title{
Review
}

\section{Adaptor Protein STAP-2 Modulates Cellular Signaling in Immune Systems}

\author{
Yuichi Sekine \\ Cellular Neuroscience, Neurodegeneration and Repair Program, \\ Departments of Neurology and Neurobiology, Yale University School of Medicine; \\ 295 Congress Ave., BCMM 433, New Haven, CT 06536, U.S.A. \\ Received May 29, 2013
}

\begin{abstract}
Signal-transducing adaptor protein-2 (STAP-2) is a recently identified adaptor protein that contains a pleckstrin homology (PH), Src homology 2 (SH2)-like domains, and proline-rich regions in its C-terminal. STAP-2 belongs to a family of STAP adaptor proteins and plays a crucial role in a variety of cellular signal transduction pathways by interacting with signaling or transcriptional molecules. STAP-2, in particular, regulates both the innate and adaptive immune systems. STAP-2 functionally interacts with signal transducers and activators of transcription 3 (STAT3) and STAT5 in cytokine signaling pathways. In addition, STAP-2 also binds to myeloid differentiation factor 88 (MyD88) and inhibitor (I) $\kappa$ B kinase $\alpha / \beta$ (IKK- $\alpha / \beta)$ in Toll-like receptor4 (TLR4) signaling, and enhances the production of inflammatory cytokines in macrophages. More importantly, experiments using STAP-2 deficient mice show that STAP-2 modulates several T-cell functions such as cell motility, survival and death. It is also reported that STAP-2 controls the immunoglobulin E (IgE)-mediated allergy response. This accumulated evidence indicates that adaptor protein STAP-2 is an important modulator of both the innate and adaptive immune systems.
\end{abstract}

Key words signal-transducing adaptor protein-2 (STAP-2); adaptor protein; cytokine; T-cell; macrophage; signal transduction

\section{INTRODUCTION}

Intracellular signaling triggered by extracellular ligands such as cytokines, hormones, and growth factors is transduced and regulated by numerous molecules. It has been well documented that the activation of receptor tyrosine kinases is mediated by oligomerization and subsequent autophosphorylation after the ligand ligation, thereby activating a downstream signaling cascade that includes kinases, transcription factors, and adaptor molecules.

In these cellular signaling pathways, adaptor proteins play an important role in modulating signal transduction. Although adaptor proteins themselves do not contain any enzymatic function, they can convey signals to their targets by interacting with other molecules through their unique domain structures. Adaptor proteins have a variety of functional domains which mediate specific protein-protein and protein-lipid interactions. ${ }^{1-5)}$ For example, Src homology 2 (SH2) and phosphotyrosine binding (PTB) domains can bind to phosphotyrosine containing motifs. ${ }^{6-9)}$ Src homology 3 (SH3) domains recognize and bind to proline-rich sequences in their respective binding partners. ${ }^{10)} \mathrm{PH}$ domains are lipid interacting modules which bind to specific phosphoinositides and play a role in recruiting proteins to defined regions of the plasma membrane. ${ }^{11)}$

This review focuses on a novel adaptor protein, signaltransducing adaptor protein-2 (STAP-2), which has recently been shown to have pleiotropic functions in cellular signaling pathways by interacting with numerous molecules through its functional domains (Table 1).

The author declares no conflict of interest.

\section{STAP FAMILY, STRUCTURE, EXPRESSION AND LOCALIZATION}

In 2000, the human STAP-2 gene was initially isolated as $b k s$, a substrate of breast tumor kinase (Brk), from a yeast two-hybrid screen from a human normal breast cDNA library, aiming to identify proteins that interact with Brk non-receptor tyrosine kinase. ${ }^{12)}$ However, the physiological functions of STAP-2/Bks had not been investigated. Three years later, the mouse homologue of STAP-2 was cloned as c-fms interacting protein by yeast two-hybrid screening of a fetal liver cDNA library, and STAP-2 gene-disrupted mice were generated to elucidate its functions. ${ }^{13)}$

STAP-2 shows high sequence and structural similarities to STAP-1, which was cloned as a c-kit-interacting protein from a hematopoietic stem cell library. ${ }^{14)}$ STAP-1 was also identified as a Tec-interacting protein, which is tyrosine phosphorylated in response to $\mathrm{B}$-cell receptor (BCR) stimulation and termed as BCR Downstream Signaling 1 (BRDG1). ${ }^{15)}$ STAP-1 and STAP-2 share conserved domains, i.e., an N-terminal $\mathrm{PH}$ domain and a region distantly related to the $\mathrm{SH} 2$ domain (referred to as an "SH2-like" domain in Fig. 1), and their overall amino acid sequence identity is $33 \%$. Sequence similarity between STAP-1 and STAP-2 is observed as follows: N-terminal $\mathrm{PH}$ domain is 36\% identical (and 58\% similar) and SH2-like domain located in the central region of the molecule is $40 \%$ identical. The SH2-like domain in STAP-2 also shares sequence similarity with phospholipase $\mathrm{C} \gamma(\mathrm{PLC} \gamma) \mathrm{SH} 2$ domain (amino acid identity is 29\%). ${ }^{13)}$ In contrast to STAP-1, STAP-2 conserves a proline-rich domain and a YXXQ motif in its C-terminus: the former is required for the binding to $\mathrm{SH} 3$ domain-containing proteins and the latter is conserved in the cytoplasmic region of certain types of cytokine receptors and is known to serve as the docking module to bind to the $\mathrm{SH} 2$ domain in signal transducer and activator of transcription 3 
Table 1. STAP-2 Interacting Proteins

\begin{tabular}{|c|c|c|c|}
\hline Interacting molecule & Domain or region & Functions & References \\
\hline BRK & PH (SH2-like) & Brk/STAT3/STAT5 signaling $\uparrow$ & $12,82,83,84$ \\
\hline $\mathrm{c}-\mathrm{Fms}$ & PH (SH2-like) & M-CSF/c-Fms signaling $\downarrow$ & $13,36,37$ \\
\hline STAT3 & YXXQ motif (SH2-like) & STAT3 phosporylation and activation $\uparrow$ & 13,83 \\
\hline STAT5a, 5b & PH, SH2-like & STAT5 phosporylation and activation $\downarrow$ & 16 \\
\hline MyD88 & SH2-like & LPS/TLR4 signaling $\uparrow$ & 27 \\
\hline $\mathrm{IKK} \alpha, \beta$ & SH2-like & LPS/TLR4 signaling $\uparrow$ & 27 \\
\hline FAK & SH2-like & T-cell adhesion $\downarrow$ & 17 \\
\hline $\mathrm{Cbl}$ & PH, SH2-like & T-cell adhesion $\downarrow$ & 17,18 \\
\hline Vav1 & SH2-like, C-terminal & T-cell migration $\uparrow$ & 52 \\
\hline Rac1 & n.d. & T-cell migration $\uparrow$ & 52 \\
\hline Fas/CD95 & n.d. & T-cell apoptosis $\uparrow$ & 64 \\
\hline Caspase- 8 & SH2-like & T-cell apoptosis $\uparrow$ & 64 \\
\hline PLC $\gamma 1,2$ & SH2-like, C-terminal & IgE/FceRI signaling $\downarrow$ & 76 \\
\hline LMP1 & PH, SH2-like & LMP1/NF- $\kappa \mathrm{B}$ signaling $\uparrow$ & 81 \\
\hline TRAF1 & n.d. & LMP1/NF- $\kappa \mathrm{B}$ signaling $\uparrow$ & 81 \\
\hline TRAF3 & PH, SH2-like & LMP1/NF- $\kappa \mathrm{B}$ signaling $\uparrow$ & 81 \\
\hline BCR-ABL & SH2-like & BCR-ABL/STAT5, ERK signaling $\uparrow$ & 85 \\
\hline
\end{tabular}

This table summarizes the STAP-2-interacting proteins. The interacting domains and the functions of STAP-2 are also shown. n.d., not determined

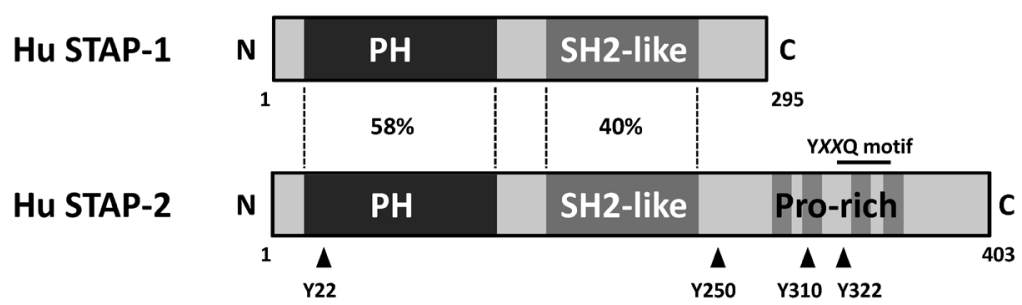

Fig. 1. Structural Comparison of STAP Family Proteins

The domain structures of human STAP family proteins are schematically shown. Four predicted tyrosine phosphorylation sites of STAP-2 are also shown. PH, Pleckstrin homology; SH2, Src homology 2; Pro-rich, Proline rich region.

(STAT3) (Fig. 1).

Western blotting analyses by Mitchell et al. showed that STAP-2 protein was detectable in various human tissues tested. ${ }^{12)}$ In contrast, STAP-1 mRNA has been shown to have hematopoietic-specific expression. ${ }^{14,15)}$ It is also shown by Minoguchi et al. that STAP-2 mRNA was inducible in some tissues or cell lines. The STAP-2 mRNA was markedly elevated at 3 to $6 \mathrm{~h}$ in mouse liver after intraperitoneal injection of lipopolysaccharide (LPS). In addition, STAP-2 mRNA expression was also strongly induced in M1 cells, a murine myeloid leukemia cell line treated with leukemia inhibitory factor, LIF. $^{13)}$ In contrast, STAP-1 mRNA expression in M1 cells was diminished after treatment with LIF. ${ }^{14)}$ Figure 2 compares the expression levels of STAP-1 and STAP-2 mRNAs in mouse, and demonstrates that STAP-1 mRNA is highly expressed in immune tissues, including the spleen and thymus, whereas STAP-2 mRNA is ubiquitously expressed, not solely in immune tissues.

Ectopically expressed STAP-2 localizes throughout the cytoplasm and nucleus in many different types of cell lines, including the human epithelial carcinoma cell line, A431, ${ }^{13)}$ African green monkey kidney cell line, Cos- $7,{ }^{16)}$ human Tcell leukemia cell line, Jurkat ${ }^{17)}$ and human cervix carcinoma cell line, HeLa. ${ }^{18)}$ In the case of A431 cells, epidermal growth factor (EGF) induces rapid (5-10 min) translocation of green fluorescent protein (GFP)-fused STAP-2 wild type (WT) protein to the plasma membrane, while mutant STAP-2 that lacks

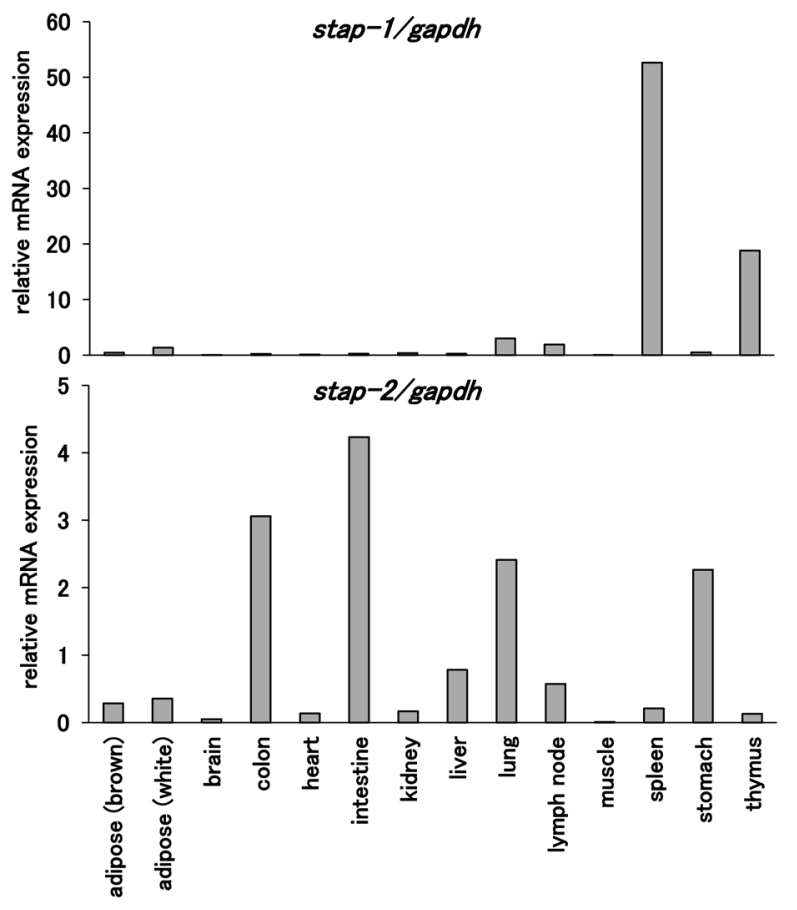

Fig. 2. Expression Profile of STAP-1 and STAP-2 mRNA in Mouse Tissues

Total RNA samples isolated from these tissues were subjected to quantitative real-time PCR analysis using STAP-1 and STAP-2 primers. Data represent the levels of these mRNA normalized to that of the GAPDH internal control. 
the PH domain fails to translocate to the plasma membrane in response to EGF. ${ }^{13)}$ Hence, the PH domain of STAP-2 is necessary for plasma membrane recruitment in response to EGF.

\section{STAP-2 REGULATES JANUS KINASE (JAK)/STAT SIGNALING PATHWAYS}

The Jak/STAT pathway is one of the major pathways in cytokine signaling. ${ }^{19-22)}$ Binding of a cytokine to its cognate receptor results in receptor dimerization and the activation of Jak tyrosine kinases. The activated Jak subsequently phosphorylates specific tyrosine residues on the cytokine receptor and serves as a docking site for STATs. Recruited STATs are tyrosine phosphorylated by activated Jak, then dimerize via SH2 domain phosphotyrosine interactions, and subsequently leave the receptor and translocate into the nucleus where they activate target gene transcription. Seven STAT proteins have been identified: STAT1, STAT2, STAT3, STAT4, STAT5A, STAT5B, and STAT6. STAT2, STAT4 and STAT6 are activated by a relatively small number of cytokines and play a distinct role in the development of T-cells and in interferon (IFN)- $\gamma$ signaling, whereas STAT1, STAT3 and STAT5 are activated by a variety ligands in several different tissues and are also involved in cell-cycle, apoptosis and tumorigenesis. ${ }^{23)}$

STAP-2 contains a YXXQ motif, which is located in its Cterminal region, is conserved among various mammals, and has been expected as a potential STAT3 binding site. Indeed, STAP-2 interacted with STAT3 through its YXXQ motif, whereas the STAP-2 Y322F mutant, in which the tyrosine residue at 322 is substituted with phenylalanine, failed to bind. ${ }^{13)}$ It should be noted that STAP-2 expression was elevated in response to interleukin (IL)-6 in primary hepatocytes. On the other hand, STAT3 activation, as well as the acute phase responses that are regulated by the IL-6/STAT3 pathway, was impaired in the liver of STAP-2 deficient mice. ${ }^{13)}$ Furthermore, transient over-expression of STAP-3 in cultured cells results in the STAT3 activation depending on its YXXQ motif. ${ }^{13)}$ It has also been shown that STAP-2 tyrosine-250 (Tyr250), a major tyrosine phosphorylation site by $\mathrm{v}-\mathrm{Src}$, Jak2 and LIF, is involved in the STAT3-enhancing activity of STAP-2, since the substitution of tyrosine residue at 250 with phenylalanine failed to enhance STAT3 transcriptional activity. ${ }^{13,24)}$ These observations demonstrate that tyrosine phosphorylation of STAP-2 is important for enhancing STAT3 transcriptional activation. In conclusion, these data indicate that STAP-2 is an adaptive protein in IL-6- and LIF-mediated STAT3 signaling.

Previously, STAP-1 has been shown to associate with STAT5 through its SH2 domain. ${ }^{14)}$ Interestingly, STAP-2 binds not only to STAT3 but also to STAT5A and STAT5B through its $\mathrm{PH}$ and $\mathrm{SH} 2$-like domains. Furthermore, the association of STAP-2 and STAT5 was attenuated by the treatment of cells with IL-2. ${ }^{16)}$ From these observations, STAP-2 is thought to interact with a non-phosphorylated form of STAT5 and to dissociate from a tyrosine-phosphorylated form of STAT5 that is activated in response to cytokine stimulation. Indeed, the level of STAT5 tyrosine phosphorylation and STAT5-dependent transcription in response to erythropoietin or IL-3 was shown to be decreased in cells that overexpress STAP-2. In addition, STAP-2 suppresses the proliferation of the mouse pro-B cell line, $\mathrm{Ba} / \mathrm{F} 3{ }^{25)}$ which is an IL-3/STAT5-dependent cell line. It has been shown that there is a profound deficiency of peripheral T-cells in STAT5A/5B double knockout mice, indicating that STAT5 proteins play an essential role in IL-2 signaling. ${ }^{26}$ Interestingly, STAP-2-deficient thymocytes show enhanced cell growth in response to IL-2. ${ }^{16}$ ) These data suggest that STAP-2 might negatively regulate STAT5-mediated cytokine signaling.

Taken together, in the Jak/STAT pathway, STAP-2 plays a role in regulating the transcriptional activity of STATs in response to a variety of cytokine stimulations. As its unique characteristics, STAP-2 shows pleiotropic function in cytokine signaling. The STAT3-mediated cytokine signaling through

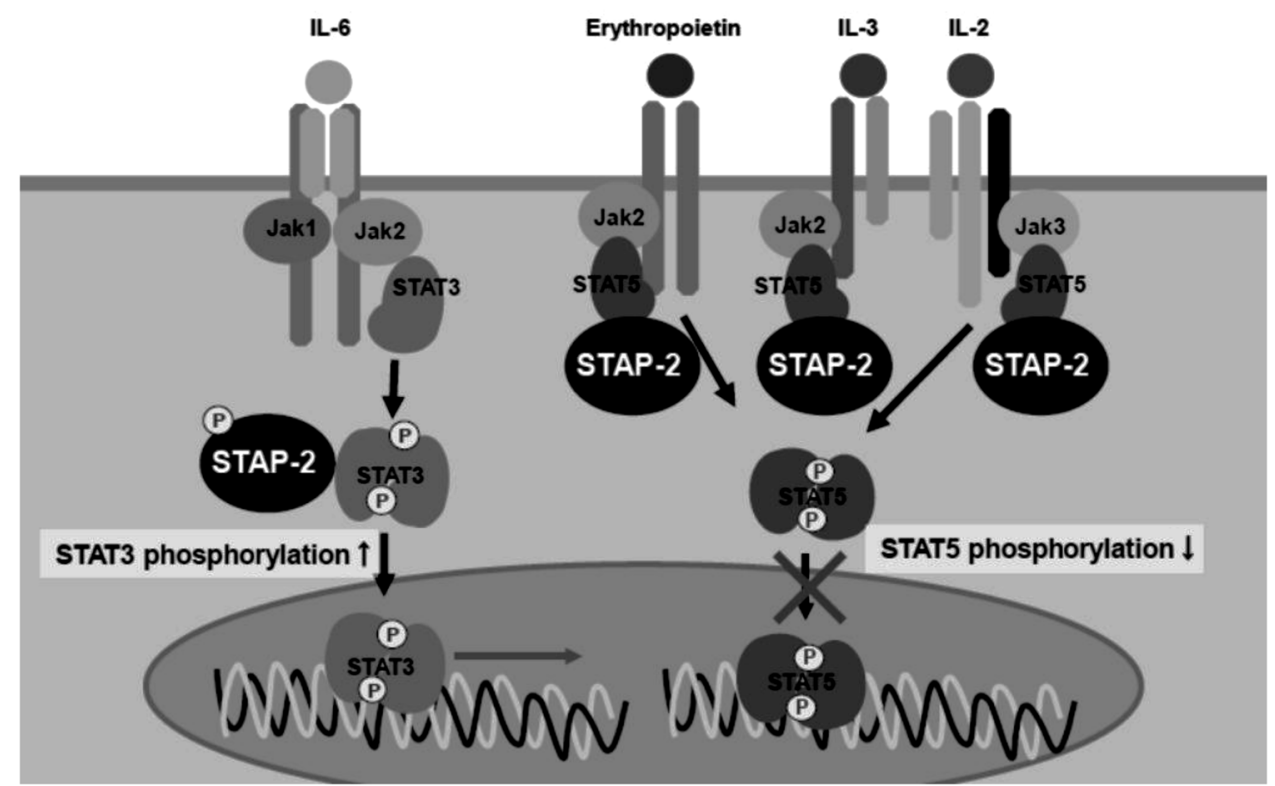

Fig. 3. STAP-2 Regulates Jak/STAT Signaling Pathways

Schematic showing the proposed function of STAP-2 in the Jak/STAT signaling pathways. STAP-2 interacts with STAT3 through the YXXQ motif in the C-terminal region and positively regulates STAT3 transcriptional activation. STAP-2 interacts with STAT5 through its PH and SH2-like domain and negatively regulates STAT5mediated signaling. 
IL-6 and LIF is enhanced by STAP-2. On the other hand, STAP-2 down-regulates the STAT5-mediated cytokine signaling pathways triggered by IL-2, IL-3 or erythropoietin (Fig. 3).

\section{STAP-2 IN MACROPHAGE}

4.1 STAP-2 Regulates Toll-Like Receptor (TLR) Signaling Pathways ${ }^{27)}$ TLRs are type-I transmembrane proteins containing leucine-rich repeat motifs in the extracellular region and a Toll/interleukin-1 receptor (TIR) domain in the intracellular region, and they play an essential role in innate immunity in organisms ranging from insects to mammals. ${ }^{28-31)}$ Microorganisms express highly conserved sequences known as pathogen-associated molecular patterns (PAMPs) which are recognized by innate immune cells such as macrophages and dendritic cells through a series of TLRs. Cell surface TLRs, including TLR1, TLR2, TLR4, TLR5, TLR6 and TLR11, recognize mainly microbial membrane components such as LPS, diacyl or triacyl lipopeptides and zymosan. On the other hand, microbial nucleic acids such as double-stranded RNA and bacterial CpG DNA, are detected by TLR3-, TLR7-, TLR8- and TLR9-expressing intracellular vesicles, in endoplasmic reticulum (ER), endosomes, lysosomes and endolysosomes. ${ }^{32-35)}$ PAMPs recognition by TLRs leads to the recruitment of various TIR domain-containing adaptors containing MyD88, TIR-associated protein (TIRAP), Toll-receptor-associated activator of interferon (TRIF) and Toll-receptor-associated molecule (TRAM), then activate subsequent signaling such as nuclear factor (NF) $-\kappa \mathrm{B}$ and mitogen-activated protein kinases (MAPK), resulting in the production of inflammatory cytokines and the expression of co-stimulatory molecules. The se- creted inflammatory cytokines, then, not only stimulate macrophages and natural killer cells that directly kill pathogens but also enhance the clonal $\mathrm{B}$ and $\mathrm{T}$ lymphocyte responses.

As mentioned above, up-regulation of STAP-2 mRNA was observed in mouse liver after intraperitoneal injection of LPS, and in LIF-treated M1 cells that underwent differentiation into macrophages. ${ }^{13)}$ The expression patterns of STAP-2 mRNA in macrophages suggest the involvement of STAP-2 in LPS signaling. Indeed, compared to WT mice, STAP-2 knock out (KO) mice showed a significantly reduced response in terms of LPS-induced TNF- $\alpha$ production in the serum. ${ }^{27)}$ In addition, macrophages isolated from STPA-2 KO mice showed lowered production of TNF- $\alpha$ and IL- 6 in response to LPS, compared with those from WT mice. ${ }^{27)}$

Phosphorylation and degradation of inhibitor (I) $\kappa \mathrm{B} \alpha$ leads to NF- $\kappa \mathrm{B}$ transcriptional activation, subsequently producing inflammatory cytokines in the LPS/TLR4 signaling pathway. In contrast to WT mice-derived macrophages, LPS-induced phosphorylation of $\mathrm{I} \kappa \mathrm{B} \alpha$ was diminished in macrophages from STAP-2-deficient mice. ${ }^{27)}$ However, activation of p38 MAP kinase (MAPK) and JNK in response to LPS was similarly observed in both STAP-2-deficient macropages and in WT ones. ${ }^{27)}$ Furthermore, a STAP-2-overexpressing macrophage cell line Raw264.7, Raw/STAP-2, produced significantly higher amounts of TNF- $\alpha$ and IL- 6 after LPS stimulation than Raw/pcDNA3, which represents parental Raw264.7 transfected with pcDNA3 vector alone. ${ }^{27)}$ It was also observed that LPSstimulated Raw/STAP-2 cells showed enhanced NF- $\kappa$ B activation. $^{27)}$

Co-immunoprecipitation experiments showed that STAP-2 interacted with MyD88, IKK $\alpha$ and IKK $\beta$ through its SH2-like domain, but not with IRAK1 and TRAF6. ${ }^{27)}$ Interestingly, the

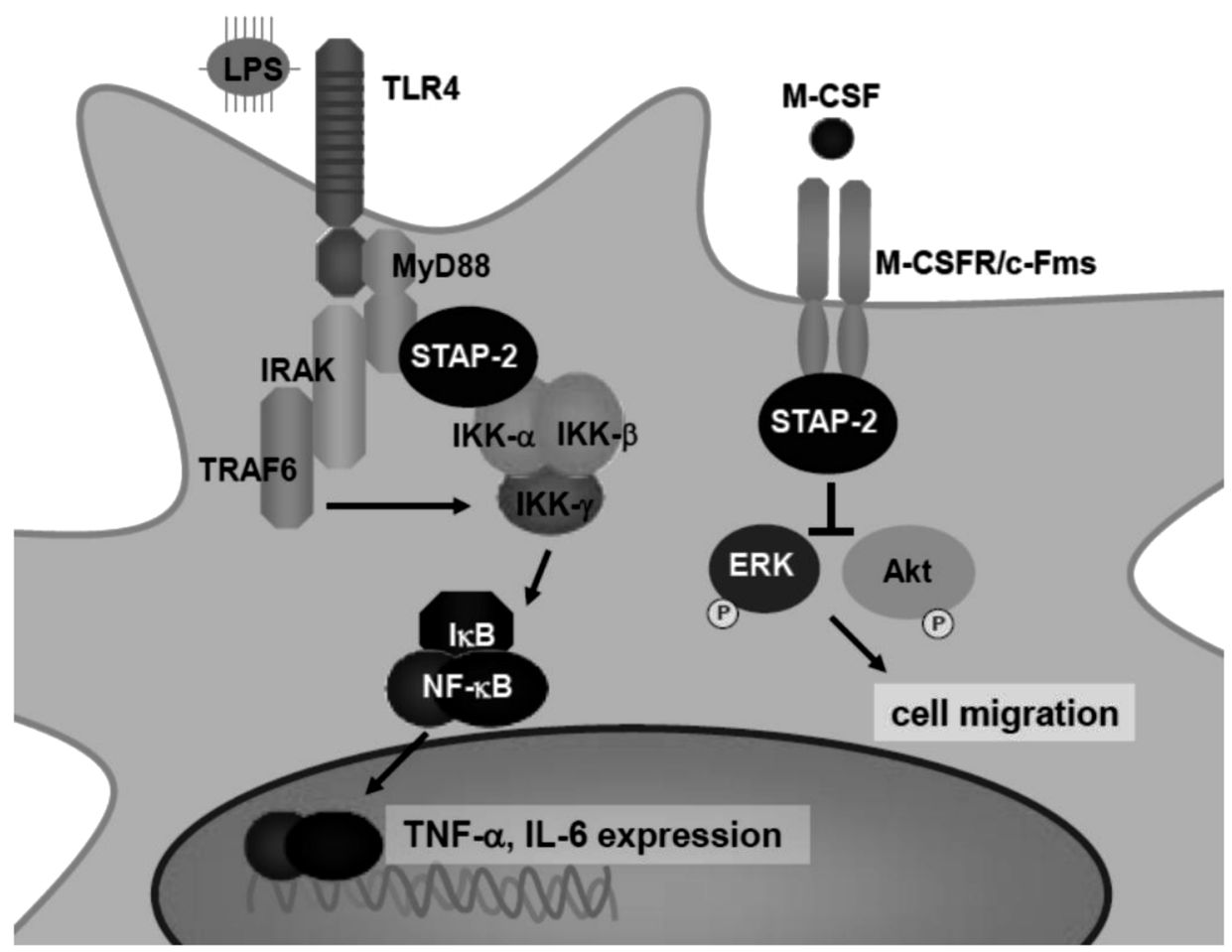

Fig. 4. STAP-2 Regulates Macrophage Functions

STAP-2 enhances the LPS/TLR4 signaling pathway. STAP-2 interacts with MyD88 and IKK- $\alpha / \beta$, leading to the enhancement of NF- $\kappa$ B activity and the production of inflammatory cytokines. STAP-2 may constitute an alternative pathway from LPS/TLR4 to NF- $\kappa$ B activation instead of the TRAF6-IRAK1 pathway. STAP-2 regulates the M-CSF/c-Fms signaling pathway STAP-2 interacts with c-Fms, leading to the suppression of ERK and Akt phosphorylation. The suppression of M-CSF/c-Fms signaling by STAP-2 results in the regulation of macrophage migration. 
association between MyD88 and IKK $\beta$ was only observed in the presence of STAP-2. ${ }^{27}$ This data suggests that STAP-2 is able to interact with both MyD88 and IKK, and plays a role in forming a molecular complex that leads to NF- $\kappa \mathrm{B}$ activation (Fig. 4).

Bacterial or virus CpG motifs are recognized by TLR9 and induce inflammatory cytokine secretion through MyD88-dependent NF- $\kappa$ B activation, whereas polyinosinic-polycytidylic acid (poly(I:C))/TLR3 signaling uses TRIF instead of MyD88 to produce inflammatory cytokines. ${ }^{28)}$ It should be noted that the production of TNF- $\alpha$ and IL-6 through the CpG/TLR9 pathway was impaired in STAP-2-deficient macrophages, while that through the poly(I:C)/TLR3 pathway remained intact. $^{27)}$ Thus, STAP-2 augments the signaling triggered by LPS/TLR4 and CpG/TLR9 mediated through MyD88, thereby stimulating cytokines production by macrophages, while it does not participate in the MyD88-independent signaling pathway.

Taken together, STAP-2 positively regulates TLR signaling by interacting with MyD88 and IKKs to activate NF- $\kappa \mathrm{B}$ and induce inflammatory cytokines in macrophage cells. Furthermore, the data suggest the possibility that STAP-2 can be considered a novel candidate for anti-inflammatory drug development to regulate the expression of NF- $\kappa \mathrm{B}$-dependent genes.

4.2 STAP-2 Regulates Macrophage-Colony Stimulating Factor (M-CSF) Receptor Signaling Pathways STAP-2 plays a role in macrophages, not only in regulating TLR signaling but also in M-CSF (macrophage-colony stimulating factor)-mediated signaling. ${ }^{36,37)} \mathrm{M}-\mathrm{CSF}$, known as CSF-1, is the principal molecule responsible for the development, differentiation, proliferation, survival and motility of macrophage lineages. ${ }^{38-40)} \mathrm{M}-\mathrm{CSF}$ binds to its receptor c-Fms which is encoded by the c-fms proto-oncogene, ${ }^{41)}$ and subsequently induces the dimerization of c-Fms, leading to the autophosphorylation of a number of tyrosine residues throughout the cytoplasmic domain of the receptor. Tyrosine-phosphorylation of c-Fms induces the recruitment of intracellular signaling molecules, resulting in activation of both the PI3K/Akt and MAPK/ERK signaling cascades. ${ }^{42-44)}$ M-CSF/PI3K signaling regulates macrophages' cytoskeletal rearrangement and motility. $^{44,45)}$

Originally, STAP-2 was cloned by yeast two-hybrid screening using $\mathrm{c}-\mathrm{fms}$ as bait. ${ }^{13)}$ Co-immunoprecipitation assays using mammalian cells revealed that STAP-2 interacts with c-Fms through its $\mathrm{PH}$ domain independently of M-CSF stimulation. ${ }^{36)} \mathrm{M}-\mathrm{CSF}$ stimulation does not affect the interaction between c-Fms and STAP-2, although M-CSF stimulation induces tyrosine-phosphorylation of STAP-2 via c-Fms. MCSF/c-Fms-mediated tyrosine-phosphorylation of STAP-2 is observed mainly at Tyr-250 and weakly at Tyr-310. Intriguingly, tyrosine-phosphorylation of c-Fms after the stimulation with M-CSF was suppressed in Raw/STAP-2 cells. ${ }^{36)}$ Furthermore, phosphorylation of Akt and ERK in response to M-CSF was also impaired in Raw/STAP-2 cells. ${ }^{36)}$ In contrast, bone marrow-derived macrophages (BMMs) isolated from STAP-2 KO mice showed enhanced phosphorylation of Akt and ERK in response to M-CSF, compared to BMMs from WT mice. ${ }^{37)}$ Thus, STAP-2 modulates M-CSF-mediated PI3K/Akt and Ras/ERK signaling pathways in macrophages. More importantly, STAP-2 regulates macrophage cell migration. When Raw/STAP-2 cells were subjected to trans-well assay, fewer numbers of cells migrated toward M-CSF, compared to Raw/ pcDNA3 cells. ${ }^{36)}$ In contrast, the wound-healing assay using STAP-2-deficient BMMs demonstrated elevated cell motility in response to $\mathrm{M}-\mathrm{CSF}{ }^{37)}$

These findings suggest that STAP-2 controls not only TLRmediated cytokine production, but also M-CSF-induced cell migration in macrophage cells (Fig. 4). It is likely that STAP-2 maintains the homeostasis of macrophage-mediated functions by regulating cytokine production and cell motility during infection or inflammatory diseases.

\section{STAP-2 IN T-CELLS}

5.1 STAP-2 Modulates T-Cell Motility In the immune system, lymphocyte trafficking and homing to specific microenvironments are very important to maintaining homeostasis and controlling the immune response. ${ }^{46-49)}$ Lymphocyte motility is mainly controlled by selectin- and integrin-mediated cell adhesion to the extracellular matrix (ECM) and chemokinemediated cell migration to specific sites. Integrins are known to regulate rolling and firm adhesion of leukocytes. ${ }^{47)}$ STAP-2 plays a role in regulating $\mathrm{T}$-cell adhesion by modulating integrin-mediated signaling. ${ }^{17)}$ It is not surprising that STAP-2 regulates the motility of T-cells, as it does in M-CSF-stimulated macrophages, because STAP-2 is expressed in T-cells, in which it participates in IL-2/STAT5-mediated cell growth. ${ }^{16)}$ Splenocytes and T-cells from STAP-2-deficient mice showed enhanced cell adhesion to fibronectin (FN), a component of ECM, after treatment with phorbol myristate acetate (PMA). ${ }^{16}$ In addition, Jurkat cells that stably over-express STAP-2 (Jurkat/STAP-2) demonstrated reduced cell adhesion to FN after integrin activation with a $\beta 1$-integrin specific antibody. In the case of STAP-2 deficient T-cells, the expression level of focal adhesion kinase (FAK) proteins was elevated. ${ }^{16)}$ On the other hand, overexpression of STAP-2 induces a dramatic decrease in the protein content of FAK in Jurkat T-cells. ${ }^{16}$ FAK is a ubiquitously expressed non-receptor protein tyrosine kinase, which has emerged as a crucial molecule for integrating signals from integrins and receptor tyrosine kinases in processes such as cell survival, proliferation and motility. ${ }^{50,51)}$ It is noteworthy that STAP-2 were shown to associate with FAK through its SH2-like domain, and the treatment of cells with proteasome inhibitors resulted in the accumulation of polyubiquitinated forms of FAK in Jurkat/STAP-2 cells. ${ }^{17)}$ Furthermore, STAP-2 can also interact with the E3-ubiquitin ligase, $\mathrm{Cbl}$, and recruits it to FAK. ${ }^{17)}$ In addition, a decrease in $\mathrm{Cbl}$ caused increased levels of FAK proteins in Jurkat/STAP-2 cells. ${ }^{17)}$ Interestingly, it is also demonstrated that STAP-2 protein expression levels are also regulated by this proteasomal degradation. ${ }^{18)}$ These lines of evidence indicate that STAP-2 is a potential novel regulator of integrin/FAK-mediated T-cell adhesion (Fig. 5).

STAP-2 regulates not only integrin-mediated T-cell adhesion but also chemokine-induced T-cell migration. ${ }^{52)}$ Chemokines are chemoattractive cytokines which bind to seventransmembrane $\mathrm{G}$ protein-coupled receptors, and are involved in chemotaxis, transendothelial migration of leukocytes during immune and inflammatory responses, non-inflammatory functions during $\mathrm{T}$ and $\mathrm{B}$ cell development, lymphocyte trafficking, and cell compartmentalization in lymphoid tissues. ${ }^{46,53,54)}$ Stromal cell-derived factor- $1 \alpha(\mathrm{SDF}-1 \alpha$; CXCL12) is a member 


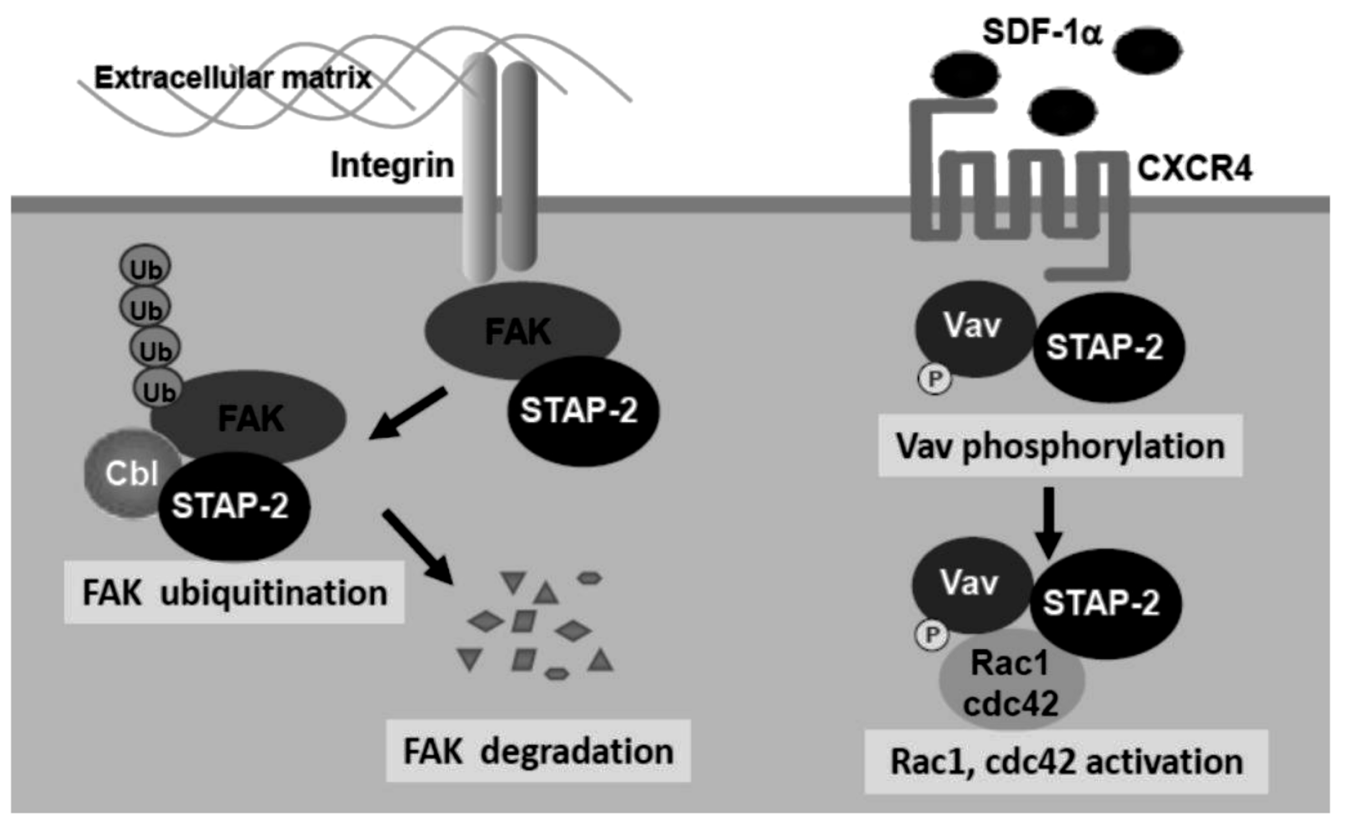

Fig. 5. STAP-2 Modulates T-Cell Functions

STAP-2 is a crucial modulator of T-cell motility. T-Cells from STAP-2 deficient mice show enhancement of integrin-mediated cell adhesion to fibronectin. STAP-2 interacts with both FAK and $\mathrm{Cbl}$ in T-cells and plays a role in the regulation of proteasome-mediated degradation of FAK by recruiting Cbl. STAP-2 also interacts with Vavl in the downstream of SDF-1 $\alpha$ mediated signaling. STAP-2 regulates SDF-1 $\alpha$-induced Vav1/Rac1 activation, controlling chemotaxis toward to SDF-1 $\alpha$ in T-cells.

of the CXC subfamily of chemokines and uses CXCR4 as the receptor. ${ }^{55-58)}$ CXCR4 is expressed on a number of cell types such as T cells, hematopoietic stem cells and progenitor cells, and mediates the signaling that leads to the development and migration of these cells. ${ }^{55-58)}$

SDF- $1 \alpha$-dependent migration of T-cells isolated from STAP-2 KO was lower than that of T-cells from WT mice. ${ }^{52)}$ In contrast, the chemotactic response of Jurkat/STAP-2 cells is elevated in response to SDF- $1 \alpha$ compared with Jurkat/ pcDNA3 cells. ${ }^{52)}$ Thus, STAP-2 can up-regulate the directional migration of $\mathrm{T}$ cells toward SDF- $1 \alpha$. ERK is not involved in STAP-2-dependent up-regulating signaling during T-cell migration, since the levels of ERK phosphorylation in Jurkat/ pcDNA3 and Jurkat/STAP-2 cells were comparable after the stimulation with SDF1- $\alpha{ }^{52)}$ It is known that SDF- $1 \alpha$-induced activation of Rho GTPases, including RhoA, Racl and Cdc42, controls T-cell migration. ${ }^{59,60)}$ Indeed, SDF- $1 \alpha$ causes robust activations of Rac1 and Cdc42 in Jurkat/STAP-2 cells, but not in Jurkat/pcDNA3 cells. ${ }^{52)}$ Furthermore, the colocalization and association of STAP-2 with Rac1 was observed after stimulation with SDF-1 $\alpha .{ }^{52)}$ Vav1, a guanine-nucleotide exchange factor (GEF), plays a critical role in T-cell development and activation. $^{61,62)}$ In the SDF-1 $\alpha$ signaling pathway, Vav1 acts as a GEF for Rac1, and inhibition of Vav1/Rac1 impairs SDF1- $\alpha$-promoted T-cell migration. ${ }^{63)}$ STAP-2 was shown to associate with Vav1 in the absence of SDF-1 $\alpha$ stimulation in T-cells, ${ }^{52}$ indicating that STAP-2 constitutively interacts with Vav1. When endogenous Vavl protein expression was lowered, binding between STAP-2 and Rac1 was abolished, thereby resulting in decreased Racl activation. ${ }^{52)}$ Furthermore, phosphorylated levels of Vav1 after SDF1- $\alpha$ stimulation are elevated in Jurkat/STAP-2 cells, but lowered in STAP-2-deficient T-cells. ${ }^{52)}$ Accumulating data suggest that STAP-2 enhances the phosphorylation of Vav1 after SDF1- $\alpha$ stimulation and subsequently recruits Racl to Vav1, resulting in augmented Racl GTPase activation and facilitation of T-cell migration (Fig. 5).
In addition, studies on STAP-2 during T-cell migration have shown that STAP-2 controls T-cell accumulation in tissues undergoing inflammation. ${ }^{52)}$

5.2 STAP-2 Regulates Apoptosis Signaling in T-Cells ${ }^{64)}$ During the course of an immune response, T-cells clonally expand after encountering the antigen presented by antigen presenting cells (APC), after which activated peripheral Tcells are removed in the termination phase. The death of these cells is important in preventing autoimmunity and maintaining T-cell homeostasis. However, a certain number of T-cells survive, forming a pool of memory $\mathrm{T}$ cells, which are specialized cells that respond rapidly to subsequent exposure to the same antigen. ${ }^{65-67)}$ Elimination of activated T-cells during the termination phase of immune responses is mediated through two mechanisms: activation-induced cell death (AICD) and activated T-cell autonomous death (ACAD). ${ }^{68)}$ AICD is mainly triggered by death receptor signaling causing programmed cell death, or apoptosis. In contrast to AICD, ACAD is also referred to as death by neglect, death by cytokine withdrawal or passive cell death.

AICD is mediated through interactions between the death receptor Fas, known as CD95, and its ligand, FasL, expressed on activated T-cells. Fas oligomerization leads to the formation of the Fas-death-inducing signaling complex (Fas-DISC) that initiates apoptosis. The Fas-DISC consists of oligomerized, probably trimerized, Fas, the adaptor molecule FADD, procaspase-8, procaspase-10 and c-FLIP. Autoproteolytic activation of pro-caspase- 8 is induced after oligomerization at the receptor complex, followed by the release of active caspase- 8 heterotetramer into cytosol for apoptotic signal propagation. ${ }^{69-71)}$

The T-cell development in STAP-2-deficient mice, assessed by the expression of CD4 and CD8, was comparable to that in WT mice. ${ }^{16)}$ In addition, expression levels of CD3 on thymocytes and splenocytes were also indistinguishable between WT and STAP-2-deficient mice. ${ }^{16)}$ AICD is known 
to be induced in WT mice by the injection of anti-CD3 antibody, which can directly stimulate $\mathrm{TCR}$, resulting in the reduced T-cell-to-B-cell ratio and increased terminal deoxynucleotidyl transferase-mediated deoxyuridine triphosphate nick-end labeling (TUNEL)-positive CD4-positive T-cells in the spleen. ${ }^{72)}$ However, neither a reduction of the T/B cell ratio nor an increase in TUNEL-positive cells was observed in the spleen of STAP-2-deficient mice subjected to anti-CD3 antibody injection. ${ }^{64)}$ Furthermore, while WT mice injected with a superantigen, SEB, displayed an elimination of $\mathrm{V} \beta 8^{+}$(superantigen responsive) $\mathrm{T}$-cells in a Fas-dependent manner, ${ }^{73)}$ no elimination of $\mathrm{V} \beta 8^{+} \mathrm{CD} 4^{+}$-responsive cells was observed in SEB-injected STAP-2 deficient mice. ${ }^{64)}$ In neither WT mice nor STAP-2 deficient mice was elimination of the $\mathrm{V} \beta 6^{+} \mathrm{CD} 4^{+}$ T-cell population (unresponsive to SEB) observed. ${ }^{64}$ These in vivo data suggest STAP-2 is involved in T-cell AICD.

The involvement of STAP-2 in AICD was confirmed by studies in vitro. Jurkat/STAP-2 cells showed significantly enhanced cell death after treatment with phytohemagglutinin (PHA), an inducer of T-cell AICD, compared to Jurkat/ pcDNA3. ${ }^{64)}$ In addition to the death receptor stimulation, i.e., Fas activation by anti-Fas antibody, TNF- $\alpha$ and TRAILinduced cell death was also enhanced in Jurkat/STAP-2 cells. $^{64)}$ In contrast, in T-cells from STAP-2-deficient mice, FasL-induced apoptosis was impaired. ${ }^{64)}$ Furthermore, the activation of caspase- 3 and -8 was significantly enhanced in Jurkat/STAP-2 cells treated with anti-Fas antibody, whereas activation of those was impaired in FasL-stimulated STAP-2 deficient T-cells. ${ }^{64)}$ Moreover, a caspase- 8 specific inhibitor suppressed Fas-induced cell death in Jurkat/STAP-2 cells, ${ }^{64)}$ suggesting that the enhanced activation of caspase- 8 is critical to increasing the susceptibility to Fas-mediated cell death.

In the FasL/Fas signaling pathway, STAP-2 was shown to interact with Fas and caspase-8, but not with FADD. ${ }^{64)}$ Interestingly, Fas-DISC complex formation after the treatment with anti-Fas antibody was dramatically augmented in STAP2-over-expressing Jurkat cells. ${ }^{64)}$ In contrast, T-cells from STAP-2-deficient mice that show suppressed activation of caspase- 8 exhibited a reduced level of Fas-DISC complex formation after Fas ligation. ${ }^{64)}$ Thus, STAP-2 is likely to enhance the Fas-DISC formation and subsequent caspase- 8 activation.

A consensus caspase- 8 cleavage site was reported to be $(\mathrm{I} / \mathrm{L} / \mathrm{V}) \mathrm{E} X \mathrm{D}{ }^{74}$ ) STAP-2 has a VEAD sequence (residues 257-260) that may be a potential caspase- 8 cleavage site. It is noteworthy that cleaved STAP-2 fragment was detected after Fas stimulation in Jurkat/STAP-2 and HUT78 cells. ${ }^{64)}$ Furthermore, a STAP-2 mutant (STAP-2 DA), in which aspartic acid residue was substituted with alanine, did not generate any Fas-mediated cleavage fragments, although it interacted with caspase-8, but not with Fas. ${ }^{64)}$ Thus, Fas-DISC formation is abolished in Jurkat/STAP-2 DA, and proteolytic cleavage of STAP-2 at Asp260 by caspase- 8 is required for the enhancement of Fas-induced apoptosis and caspase- 8 enzymatic activation. In summary, these data indicate that STAP-2 is a novel participant in the regulation of T-cell AICD by controlling Fas-mediated caspase-8 activation (Fig. 6).

\section{STAP-2 MODULATES ALLERGIC RESPONSE}

STAP-2 regulates immune and inflammatory responses through interactions with a variety of signaling or transcrip-

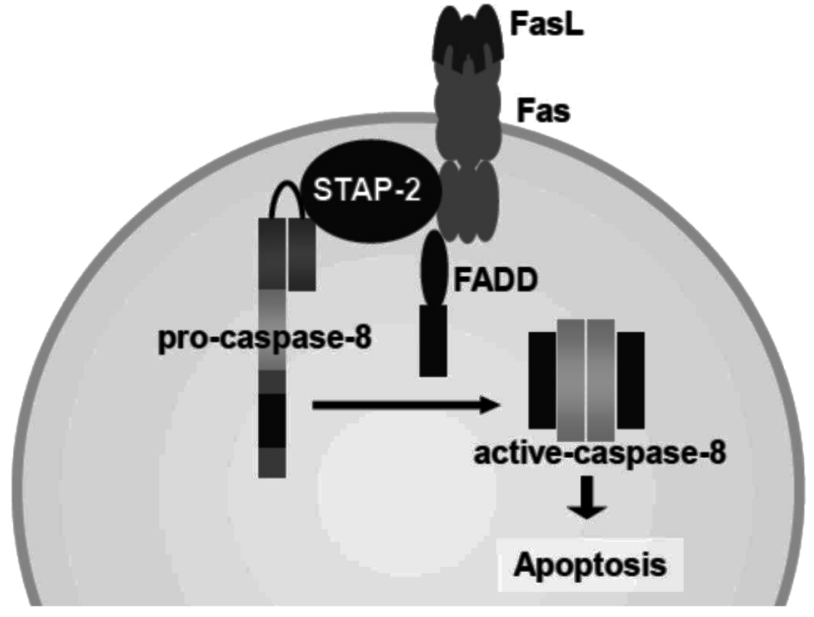

Fig. 6. STAP-2 Regulates Fas-Induced Cell Death Signaling

T-Cells from STAP-2 deficient mice show resistance to Fas-mediated apoptosis. STAP-2 interacts with both Fas and caspase- 8 but not FADD in T-cells. STAP-2 increases Fas-DISC complex formation and results in the activation of caspase- 8 and subsequent induction of apoptosis.

tional molecules. ${ }^{75)}$ It is also reported that the overexpression of STAP-2 in a rat basophilic leukemia cell line, RBL-2H3, resulted in a dramatic suppression of tyrosine phosphorylation of PLC- $\gamma$, calcium mobilization, and degranulation, all of which were triggered by immunoglobulin E (IgE)/FceRI, a high-affinity receptor for IgE. ${ }^{76)}$ These facts suggest that STAP-2 may have the potential to negatively regulate FceRImediated signals. IgE/FceRI signaling plays a central role in allergic diseases. ${ }^{77-80)}$ In mast cells and basophils, the aggregation of FceRI eventually leads to the release of granule components such as histamine and cytokines. Although further experiments using STAP-2-deficient mast cells or basophils are required to demonstrate physiological STAP-2 functions in IgE/FceRI-mediated pathogenesis of allergic diseases, STAP-2 has a potential to regulate allergic signaling and is a possible target for allergic disorder drug development.

\section{CONCLUSION}

STAP-2 expression is detected in numerous tissues and cell lines such as lymphocytes, macrophages, hepatocytes and breast tumor cell lines, and its patterns of inducible expression imply that it is a multifunctional protein. Specifically, STAP-2 regulates not only immune systems, but also controls other signal transduction pathways involved in leukemia and breast tumor cells. ${ }^{81-85)}$ Indeed, the constitutive expression of STAP-2 mRNA is observed in macrophages and T-cells, where STAP-2 regulates cellular functions. Furthermore, the induction of STAP-2 mRNA was detected in various types of cells treated with corresponding stimulatory factors; e.g., hepatocytes treated with IL- $6,{ }^{13)}$ M1 cells subjected to LIF stimulation, ${ }^{13)}$ and Epstein-Barr virus (EBV)-positive human $\mathrm{B}$ cells in parallel with latent membrane protein 1 (LMP1) expression. $^{23)}$

In these stimulatory signaling pathways, STAP-2 plays a role as an important modulator. Importantly, STAP-2 functions are regulated by tyrosine-phosphorylation, proteasomedependent degradation and caspase-8-dependent cleavage.

Recently, it has been reported that STAP-1 expression is induced in pro-inflammatory activated microglia and mac- 
rophages. Additionally, cell migration and M-CSF-induced chemotaxis is inhibited in STAP-1-expressing microglia cells. Furthermore, STAP-1 increases microglial phagocytosis, NO (nitric oxide) secretion and neurotoxicity. ${ }^{86}$ ) These findings are interesting because STAP-2 regulates M-CSF-induced macrophage migration similar to STAP-1's actions in microglia. In fact, STAP-2 mRNA is expressed in the brain, especially the cerebellum (data not shown). This may point to a role of STAP-2 in the brain. Further experiments are necessary to understand the function of STAP family proteins in the central and peripheral nervous systems.

Accumulating data suggest that a novel adaptor protein, STAP-2, is an important modulator not only for the innate immune system, as well as and the adaptive one, but also for tumorigenic signaling.

Acknowledgments The author is very grateful to professor Tadashi Matsuda for his invaluable guidance and suggestions. I also would like to express many thanks to my colleagues and collaborators for their support of my research. I thank Zoe A. Klein for kindly helping to edit the language of this manuscript.

\section{REFERENCES}

1) Koretzky GA, Myung PS. Positive and negative regulation of Tcell activation by adaptor proteins. Nat. Rev. Immunol., 1, 95-107 (2001).

2) Jordan MS, Singer AL, Koretzky GA. Adaptors as central mediators of signal transduction in immune cells. Nat. Immunol., 4, 110-116 (2003).

3) Simeoni L, Kliche S, Lindquist J, Schraven B. Adaptors and linkers in T and B cells. Curr. Opin. Immunol., 16, 304-313 (2004).

4) Massie C, Mills IG. The developing role of receptors and adaptors. Nat. Rev. Cancer, 6, 403-409 (2006).

5) Csiszár A. Structural and functional diversity of adaptor proteins involved in tyrosine kinase signalling. Bioessays, 28, 465-479 (2006).

6) Sadowski I, Stone JC, Pawson T. A noncatalytic domain conserved among cytoplasmic protein-tyrosine kinases modifies the kinase function and transforming activity of Fujinami sarcoma virus P130gag-fps. Mol. Cell. Biol., 6, 4396-4408 (1986).

7) Liu BA, Jablonowski K, Raina M, Arce M, Pawson T, Nash PD. The human and mouse complement of SH2 domain proteins-establishing the boundaries of phosphotyrosine signaling. Mol. Cell, 22, 851-868 (2006).

8) O’Bryan JP, Martin CB, Songyang Z, Cantley LC, Der CJ. Binding specificity and mutational analysis of the phosphotyrosine binding domain of the brain-specific adaptor protein ShcC. J. Biol. Chem., 271, 11787-11791 (1996).

9) Uhlik MT, Temple B, Bencharit S, Kimple AJ, Siderovski DP, Johnson GL. Structural and evolutionary division of phosphotyrosine binding (PTB) domains. J. Mol. Biol., 345, 1-20 (2005).

10) Mayer BJ, Eck MJ. SH3 domains. Minding your p's and q's. Curr. Biol., 5, 364-367 (1995).

11) Cozier GE, Carlton J, Bouyoucef D, Cullen PJ. Membrane targeting by pleckstrin homology domains. Curr. Top. Microbiol. Immunol., 282, 49-88 (2004).

12) Mitchell PJ, Sara EA, Crompton MR. A novel adaptor-like protein which is a substrate for the non-receptor tyrosine kinase, BRK. Oncogene, 19, 4273-4282 (2000).

13) Minoguchi M, Minoguchi S, Aki D, Joo A, Yamamoto T, Yumioka T, Matsuda T, Yoshimura A. STAP-2/BKS, an adaptor/docking protein, modulates STAT3 activation in acute-phase response through its YXXQ motif. J. Biol. Chem., 278, 11182-11189 (2003).

14) Masuhara M, Nagao K, Nishikawa M, Sasaki M, Yoshimura A, Osawa M. Molecular cloning of murine STAP-1, the stem-cellspecific adaptor protein containing $\mathrm{PH}$ and $\mathrm{SH} 2$ domains. Biochem. Biophys. Res. Commun., 268, 697-703 (2000).

15) Ohya K, Kajigaya S, Kitanaka A, Yoshida K, Miyazato A, Yamashita Y, Yamanaka T, Ikeda U, Shimada K, Ozawa K, Mano H. Molecular cloning of a docking protein, BRDG1, that acts downstream of the Tec tyrosine kinase. Proc. Natl. Acad. Sci. U.S.A., 96, 11976-11981 (1999).

16) Sekine Y, Yamamoto T, Yumioka T, Sugiyama K, Tsuji S, Oritani K, Shimoda K, Minoguchi M, Yoshimura A, Matsuda T. Physical and functional interactions between STAP-2/BKS and STAT5. $J$. Biol. Chem., 280, 8188-8196 (2004).

17) Sekine Y, Tsuji S, Ikeda O, Sugiyma K, Oritani K, Shimoda K, Muromoto R, Ohbayashi N, Yoshimura A, Matsuda T. Signal-transducing adaptor protein-2 regulates integrin-mediated $\mathrm{T}$ cell adhesion through protein degradation of focal adhesion kinase. J. Immunol., 179, 2397-2407 (2007).

18) Sekine Y, Yamamoto C, Ikeda O, Muromoto R, Nanbo A, Oritani K, Yoshimura A, Matsuda T. The protein content of an adaptor protein, STAP-2 is controlled by E3 ubiquitin ligase Cbl. Biochem. Biophys. Res. Commun., 384, 187-192 (2009).

19) Kishimoto T, Taga T, Akira S. Cytokine signal transduction. Cell, 76, 253-262 (1994).

20) Darnell JE Jr. STATs and gene regulation. Science, 277, 1630-1635 (1997).

21) Shuai K, Liu B. Regulation of JAK-STAT signalling in the immune system. Nat. Rev. Immunol., 3, 900-911 (2003).

22) O'Shea JJ, Holland SM, Staudt LM. JAKs and STATs in immunity, immunodeficiency, and cancer. N. Engl. J. Med., 368, 161-170 (2013).

23) Bowman T, Garcia R, Turkson J, Jove R. STATs in oncogenesis. Oncogene, 19, 2474-2488 (2000).

24) Sekine Y, Tsuji S, Ikeda O, Kakisaka M, Sugiyama K, Yoshimura A, Matsuda T. Leukemia inhibitory factor-induced phosphorylation of STAP-2 on tyrosine-250 is involved in its STAT3-enhancing activity. Biochem. Biophys. Res. Commun., 356, 517-522 (2007).

25) Mui AL, Wakao H, Kinoshita T, Kitamura T, Miyajima A. Suppression of interleukin-3-induced gene expression by a C-terminal truncated Stat5: role of Stat5 in proliferation. EMBO J., 15, 2425-2433 (1996).

26) Moriggl R, Topham DJ, Teglund S, Sexl V, McKay C, Wang D, Hoffmeyer A, van Deursen J, Sangster MY, Bunting KD, Grosveld GC, Ihle JN. Stat5 is required for IL-2-induced cell cycle progression of peripheral T cells. Immunity, 10, 249-259 (1999).

27) Sekine Y, Yumioka T, Yamamoto T, Muromoto R, Imoto S, Sugiyama K, Oritani K, Shimoda K, Minoguchi M, Akira S, Yoshimura A, Matsuda T. Modulation of TLR4 signaling by a novel adaptor protein signal-transducing adaptor protein-2 in macrophages. $J$. Immunol., 176, 380-389 (2006).

28) Akira S, Takeda K. Toll-like receptor signalling. Nat. Rev. Immunol., 4, 499-511 (2004).

29) Beutler B. Inferences, questions and possibilities in Toll-like receptor signalling. Nature, 430, 257-263 (2004).

30) Takeda K, Kaisho T, Akira S. Toll-like receptors. Annu. Rev. Immunol., 21, 335-376 (2003).

31) Yamamoto M, Takeda K, Akira S. TIR domain-containing adaptors define the specificity of TLR signaling. Mol. Immunol., 40, 861-868 (2004).

32) Latz E, Schoenemeyer A, Visintin A, Fitzgerald KA, Monks BG, Knetter CF, Lien E, Nilsen NJ, Espevik T, Golenbock DT. TLR9 signals after translocating from the ER to CpG DNA in the lysosome. Nat. Immunol., 5, 190-198 (2004).

33) Nishiya T, DeFranco AL. Ligand-regulated chimeric receptor approach reveals distinctive subcellular localization and signaling 
properties of the Toll-like receptors. J. Biol. Chem., 279, 1900819017 (2004).

34) Matsumoto M, Funami K, Tanabe M, Oshiumi H, Shingai M, Seto Y, Yamamoto A, Seya T. Subcellular localization of Toll-like receptor 3 in human dendritic cells. J. Immunol., 171, 3154-3162 (2003).

35) Kawai $T$, Akira $S$. The role of pattern-recognition receptors in innate immunity: update on Toll-like receptors. Nat. Immunol., 11, 373-384 (2010)

36) Ikeda O, Sekine Y, Kakisaka M, Tsuji S, Muromoto R, Ohbayashi N, Oritani K, Yoshimura A, Matsuda T. STAP-2 regulates cFms/M-CSF receptor signaling in murine macrophage Raw264.7 cells. Biochem. Biophys. Res. Commun., 358, 931-937 (2007).

37) Ikeda O, Sekine Y, Muromoto R, Ohbayashi N, Yoshimura A, Matsuda T. Enhanced c-Fms/M-CSF receptor signaling and woundhealing process in bone marrow-derived macrophages of signaltransducing adaptor protein-2 (STAP-2) deficient mice. Biol. Pharm. Bull., 31, 1790-1793 (2008).

38) Pixley FJ, Stanley ER. CSF-1 regulation of the wandering macrophage: complexity in action. Trends Cell Biol., 14, 628-638 (2004).

39) Hamilton JA. Colony-stimulating factors in inflammation and autoimmunity. Nat. Rev. Immunol., 8, 533-544 (2008).

40) Vairo G, Hamilton JA. Signalling through CSF receptors. Immunol. Today, 12, 362-369 (1991).

41) Sherr CJ, Rettenmier CW, Sacca R, Roussel MF, Look AT, Stanley ER. The c-fms proto-oncogene product is related to the receptor for the mononuclear phagocyte growth factor, CSF-1. Cell, 41, 665-676 (1985).

42) Valledor AF, Comalada M, Xaus J, Celada A. The differential timecourse of extracellular-regulated kinase activity correlates with the macrophage response toward proliferation or activation. J. Biol. Chem., 275, 7403-7409 (2000).

43) Kelley TW, Graham MM, Doseff AI, Pomerantz RW, Lau SM, Ostrowski MC, Franke TF, Marsh CB. Macrophage colony-stimulating factor promotes cell survival through Akt/protein kinase B. J. Biol. Chem., 274, 26393-26398 (1999).

44) Vanhaesebroeck B, Jones GE, Allen WE, Zicha D, Hooshmand-Rad R, Sawyer C, Wells C, Waterfield MD, Ridley AJ. Distinct PI(3)Ks mediate mitogenic signalling and cell migration in macrophages. Nat. Cell Biol., 1, 69-71 (1999).

45) Yeung YG, Wang Y, Einstein DB, Lee PS, Stanley ER. Colonystimulating factor-1 stimulates the formation of multimeric cytosolic complexes of signaling proteins and cytoskeletal components in macrophages. J. Biol. Chem., 273, 17128-17137 (1998).

46) Sallusto F, Mackay CR, Lanzavecchia A. The role of chemokine receptors in primary, effector, and memory immune responses. Annu. Rev. Immunol., 18, 593-620 (2000).

47) Kinashi T. Intracellular signalling controlling integrin activation in lymphocytes. Nat. Rev. Immunol., 5, 546-559 (2005).

48) Luster AD, Alon R, von Andrian UH. Immune cell migration in inflammation: present and future therapeutic targets. Nat. Immunol., 6, 1182-1190 (2005).

49) Ley K, Laudanna C, Cybulsky MI, Nourshargh S. Getting to the site of inflammation: the leukocyte adhesion cascade updated. Nat. Rev. Immunol., 7, 678-689 (2007).

50) Mitra SK, Hanson DA, Schlaepfer DD. Focal adhesion kinase: in command and control of cell motility. Nat. Rev. Mol. Cell Biol., 6, 56-68 (2005).

51) Schaller MD. Biochemical signals and biological responses elicited by the focal adhesion kinase. Biochim. Biophys. Acta, 1540, 1-21 (2001).

52) Sekine Y, Ikeda O, Tsuji S, Yamamoto C, Muromoto R, Nanbo A, Oritani K, Yoshimura A, Matsuda T. Signal-transducing adaptor protein-2 regulates stromal cell-derived factor-1 alpha-induced chemotaxis in T cells. J. Immunol., 183, 7966-7974 (2009).

53) Rossi D, Zlotnik A. The biology of chemokines and their receptors. Annu. Rev. Immunol., 18, 217-242 (2000).
54) Mackay CR. Chemokines: immunology's high impact factors. Nat. Immunol., 2, 95-101 (2001).

55) Bleul CC, Farzan M, Choe H, Parolin C, Clark-Lewis I, Sodroski J, Springer TA. The lymphocyte chemoattractant SDF-1 is a ligand for LESTR/fusin and blocks HIV-1 entry. Nature, 382, 829-833 (1996).

56) Oberlin E, Amara A, Bachelerie F, Bessia C, Virelizier JL, Arenzana-Seisdedos F, Schwartz O, Heard JM, Clark-Lewis I, Legler DF, Loetscher M, Baggiolini M, Moser B. The CXC chemokine SDF-1 is the ligand for LESTR/fusin and prevents infection by T-cell-lineadapted HIV-1. Nature, 382, 833-835 (1996).

57) Nagasawa T, Nakajima T, Tachibana K, Iizasa H, Bleul CC, Yoshie O, Matsushima K, Yoshida N, Springer TA, Kishimoto T. Molecular cloning and characterization of a murine pre-B-cell growth-stimulating factor/stromal cell-derived factor 1 receptor, a murine homo$\log$ of the human immunodeficiency virus 1 entry coreceptor fusin. Proc. Natl. Acad. Sci. U.S.A., 93, 14726-14729 (1996).

58) Klein RS, Rubin JB. Immune and nervous system CXCL12 and CXCR4: parallel roles in patterning and plasticity. Trends Immunol., 25, 306-314 (2004).

59) del Pozo MA, Vicente-Manzanares M, Tejedor R, Serrador JM, Sánchez-Madrid F. Rho GTPases control migration and polarization of adhesion molecules and cytoskeletal ERM components in T lymphocytes. Eur. J. Immunol., 29, 3609-3620 (1999).

60) Patrussi L, Baldari CT. Intracellular mediators of CXCR4-dependent signaling in T cells. Immunol. Lett., 115, 75-82 (2008).

61) Turner M, Billadeau DD. VAV proteins as signal integrators for multi-subunit immune-recognition receptors. Nat. Rev. Immunol., 2 , 476-486 (2002).

62) Tybulewicz VL. Vav-family proteins in T-cell signalling. Curr. Opin. Immunol., 17, 267-274 (2005).

63) García-Bernal D, Wright N, Sotillo-Mallo E, Nombela-Arrieta C, Stein JV, Bustelo XR, Teixido J. Vav1 and Rac control chemokine-promoted $\mathrm{T}$ lymphocyte adhesion mediated by the integrin alpha4beta1. Mol. Biol. Cell, 16, 3223-3235 (2005).

64) Sekine Y, Yamamoto C, Kakisaka M, Muromoto R, Kon S, Ashitomi D, Fujita N, Yoshimura A, Oritani K, Matsuda T. Signal-transducing adaptor protein-2 modulates Fas-mediated T cell apoptosis by interacting with caspase-8. J. Immunol., 188, 6194-6204 (2012).

65) Abbas AK. Die and let live: eliminating dangerous lymphocytes. Cell, 84, 655-657 (1996).

66) Krueger A, Fas SC, Baumann S, Krammer PH. The role of CD95 in the regulation of peripheral T-cell apoptosis. Immunol. Rev., 193, 58-69 (2003).

67) Strasser A, Pellegrini M. T-Lymphocyte death during shutdown of an immune response. Trends Immunol., 25, 610-615 (2004).

68) Brenner D, Krammer PH, Arnold R. Concepts of activated T cell death. Crit. Rev. Oncol. Hematol., 66, 52-64 (2008).

69) Krammer PH. CD95's deadly mission in the immune system. $\mathrm{Na}$ ture, 407, 789-795 (2000).

70) Hildeman DA, Zhu Y, Mitchell TC, Kappler J, Marrack P. Molecular mechanisms of activated T cell death in vivo. Curr. Opin. Immunol., 14, 354-359 (2002).

71) Krammer PH, Arnold R, Lavrik IN. Life and death in peripheral T cells. Nat. Rev. Immunol., 7, 532-542 (2007).

72) Baumann S, Dostert A, Novac N, Bauer A, Schmid W, Fas SC, Krueger A, Heinzel T, Kirchhoff S, Schutz G, Krammer PH. Glucocorticoids inhibit activation-induced cell death (AICD) via direct DNA-dependent repression of the CD95 ligand gene by a glucocorticoid receptor dimer. Blood, 106, 617-625 (2005).

73) Strasser A, Harris AW, Huang DC, Krammer PH, Cory S. Bcl-2 and Fas/APO-1 regulate distinct pathways to lymphocyte apoptosis. EMBO J., 14, 6136-6147 (1995).

74) Thornberry NA, Rano TA, Peterson EP, Rasper DM, Timkey T, Garcia-Calvo M, Houtzager VM, Nordstrom PA, Roy S, Vaillancourt JP, Chapman KT, Nicholson DW. A combinatorial approach defines specificities of members of the caspase family and gran- 
zyme B. Functional relationships established for key mediators of apoptosis. J. Biol. Chem., 272, 17907-17911 (1997).

75) Sekine Y. Novel adaptor protein, STAP-2 functions as a signal modulator in immune system. Yakugaku Zasshi, 130, 769-775 (2010).

76) Yamamoto T, Yumioka T, Sekine Y, Sato N, Minoguchi M, Yoshimura A, Matsuda T. Regulation of FcepsilonRI-mediated signaling by an adaptor protein STAP-2/BSK in rat basophilic leukemia RBL-2H3 cells. Biochem. Biophys. Res. Commun., 306, 767-773 (2003).

77) Schwartz LB. Mast cells: function and contents. Curr. Opin. Immunol., 6, 91-97 (1994).

78) Kawakami T, Galli SJ. Regulation of mast-cell and basophil function and survival by IgE. Nat. Rev. Immunol., 2, 773-786 (2002).

79) Turner H, Kinet JP. Signalling through the high-affinity IgE receptor Fc epsilonRI. Nature, 402 (Suppl.), B24-B30 (1999).

80) Kraft S, Kinet JP. New developments in FcepsilonRI regulation, function and inhibition. Nat. Rev. Immunol., 7, 365-378 (2007).

81) Ikeda O, Sekine Y, Yasui T, Oritani K, Sugiyma K, Muromoto R, Ohbayashi N, Yoshimura A, Matsuda T. STAP-2 negatively regulates both canonical and noncanonical NF-kappaB activation induced by Epstein-Barr virus-derived latent membrane protein 1 .
Mol. Cell. Biol., 28, 5027-5042 (2008).

82) Ikeda O, Miyasaka Y, Sekine Y, Mizushima A, Muromoto R, Nanbo A, Yoshimura A, Matsuda T. STAP-2 is phosphorylated at tyrosine-250 by Brk and modulates Brk-mediated STAT3 activation. Biochem. Biophys. Res. Commun., 384, 71-75 (2009).

83) Ikeda O, Sekine Y, Mizushima A, Nakasuji M, Miyasaka Y, Yamamoto C, Muromoto R, Nanbo A, Oritani K, Yoshimura A, Matsuda T. Interactions of STAP-2 with Brk and STAT3 participate in cell growth of human breast cancer cells. J. Biol. Chem., 285, 3809338103 (2010).

84) Ikeda O, Mizushima A, Sekine Y, Yamamoto C, Muromoto R, Nanbo A, Oritani K, Yoshimura A, Matsuda T. Involvement of STAP-2 in Brk-mediated phosphorylation and activation of STAT5 in breast cancer cells. Cancer Sci., 102, 756-761 (2011).

85) Sekine Y, Ikeda O, Mizushima A, Ueno Y, Muromoto R, Yoshimura A, Kanakura Y, Oritani K, Matsuda T. STAP-2 interacts with and modulates BCR-ABL-mediated tumorigenesis. Oncogene, 31, 4384-4396 (2012).

86) Stoecker K, Weigelt K, Ebert S, Karlstetter M, Walczak Y, Langmann T. Induction of STAP-1 promotes neurotoxic activation of microglia. Biochem. Biophys. Res. Commun., 379, 121-126 (2009). 